\title{
Graphene Oxide of Extra High Oxidation: A Wafer for
}

\section{Loading Guest Molecules}

\section{Appendix 1 "Graphene Oxide Characterisation"}

\author{
Abdelsattar O. E. Abdelhalima, Vladimir V.Sharoyko ${ }^{a, c, d^{*}}$, Sergei V. Ageeve,a, Vladimir S. \\ Farafonove, Dmitry A. Nerukhf, Viktor N. Postnov ${ }^{a}$, Andrey V. Petrov ${ }^{a}$, Konstantin N. \\ Semenov ${ }^{c, a, d^{*}}$
}

${ }^{a}$ Institute of Chemistry, Saint Petersburg State University, 26 Universitetskii prospekt, Saint Petersburg, 198504, Russia

${ }^{b}$ Environmental Research Department, National Center for Social and Criminological Research (NCSCR), Giza 11561, Egypt

${ }^{c}$ Pavlov First Saint Petersburg State Medical University, 6-8 L'va Tolstogo ulitsa, Saint Petersburg, 197022, Russia

${ }^{d}$ A. M. Granov Russian Research Centre for Radiology and Surgical Technologies, 70 Leningradskaya ulitsa, Saint Petersburg, 197758, Russia

${ }^{e}$ V. N. Karazin Kharkiv National University, 4 Svobody ploshchad', Kharkiv, 61022, Ukraine

fDepartment of Mathematics, Aston University, Birmingham, B4 7ET, The United Kingdom

*Corresponding authors at: 6-8 L'va Tolstogo ulitsa, Saint Petersburg, 197022, Russia (K. N. Semenov) and 26 Universitetskii prospekt, Saint Petersburg, 198504, Russia (V. V. Sharoyko). E-mail addresses: knsemenov@gmail.com (K. N. Semenov), sharoyko@gmail.com (V. V. Sharoyko). 


\subsection{GO structure analysis}

Solid-state ${ }^{13} \mathrm{C}$ NMR spectra were measured using Bruker Avance III $400 \mathrm{WB}$; operating frequency $100.64 \mathrm{MHz}$ for ${ }^{13} \mathrm{C}$ nuclei, Bruker, Massachusetts, United States.

Rigaku “MiniFlex II” X-ray powder diffractometer (Japan) was used for XRD analysis.

The thermal stability of GO was measured using NETZSCH TG 209 F1 Libra thermogravimetric analyser (Germany) in the temperature range $40-1025^{\circ} \mathrm{C}$, using pure nitrogen and the heating rate of $5^{\circ} \mathrm{C} \mathrm{min}^{-1}$.

IR spectra were measured by FT-IR spectrometer Nicolet 8700 (USA).

Raman spectra were obtained using Raman spectrometer Horiba Jobin-Yvon LabRam HR800 (Japan).

UV/Vis spectra were recorded using SOLAR CM 2203 spectrofluorometer (Solar, CJSC, Minsk, Belarus) in the range of wavelengths $\lambda=200-800 \mathrm{~nm}$.

The morphology of GO was determined using scanning electron microscope (SEM) JSM7001F (Japan) and high-resolution transmission electron microscope (HRTEM) Zeiss Libra 200FE (Germany).

\subsection{Adsorption experiment}

To determine the specific surface area and to study the porous structure the Micromeritics ASAP 2020MP device (USA) was applied. Sorption characteristics were studied using nitrogen as an adsorbate at $T=77.4 \mathrm{~K}$.

\subsection{Size distribution and $\zeta$-potentials in aqueous solution}

The size distribution and $\zeta$-potential of GO particles in solutions $\left(C=1.8-9.0 \mathrm{~g} \cdot \mathrm{l}^{-1}\right)$ were obtained using Malvern Zetasizer 3000 (Malvern Instruments, Malvern, United Kingdom) spectrometer at $T=298.15 \mathrm{~K}$. The values of the polydispersity index were equal to $0.24-0.27$. Smoluchowski equation was applied for the calculation of $\zeta$-potentials. 


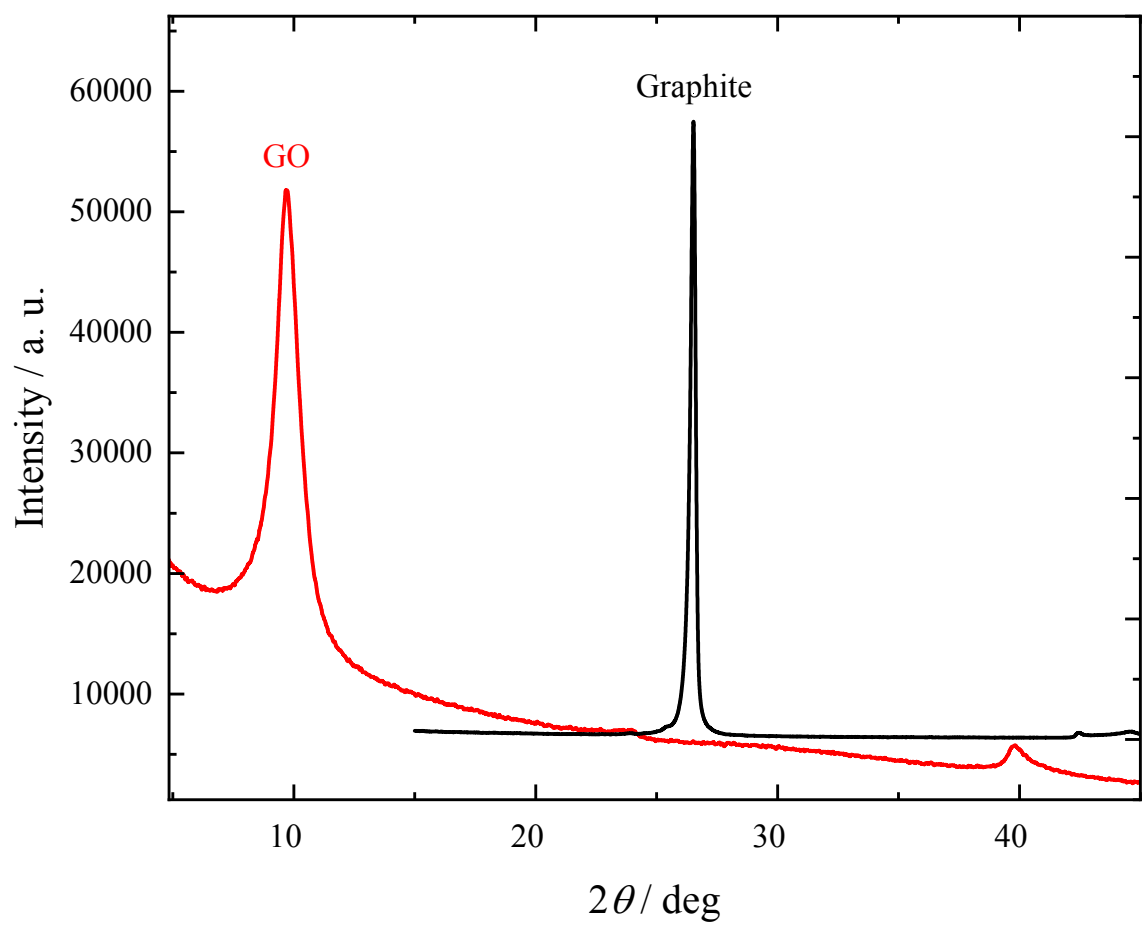

Fig. S1. X-ray diffraction spectra of graphite (-) and GO (-). 


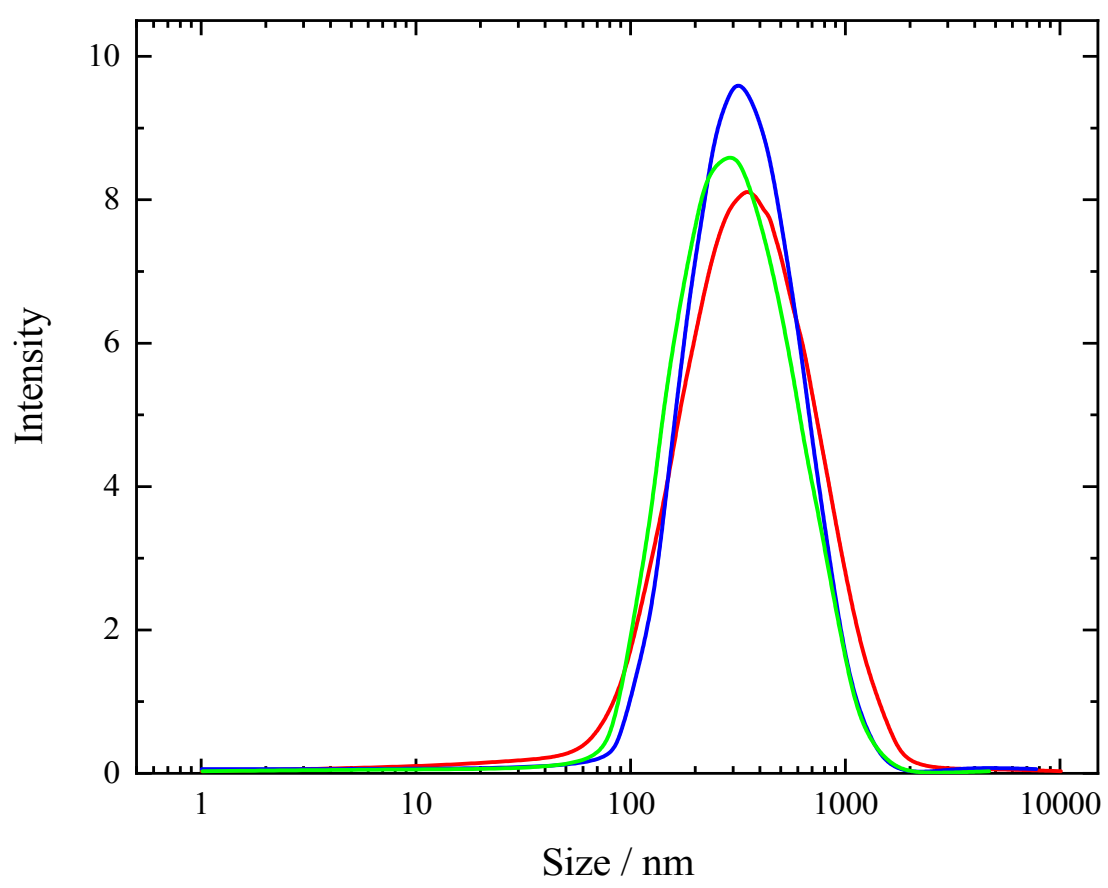

Fig. S2. Size distribution of GO particles in the aqueous dispersion $\left(C=9 \mathrm{~g} \cdot \mathrm{l}^{-1}\right)$. 


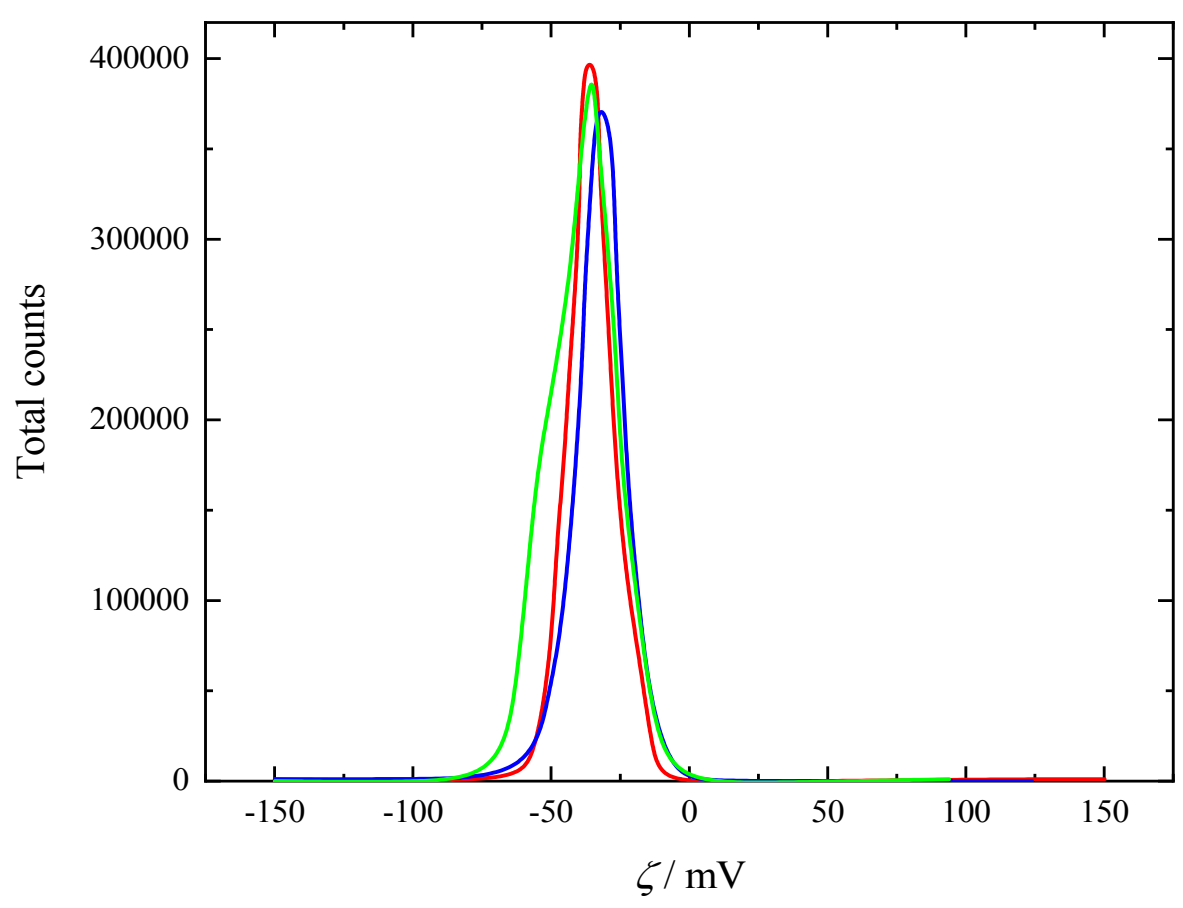

Fig. S3. $\zeta$-Potential distribution of GO particles in the aqueous dispersion $\left(C=9 \mathrm{~g} \cdot \mathrm{1}^{-1}\right)$. 